\title{
Fungal infections and antifungal prophylaxis in pediatric cardiac extracorporeal life support
}

\author{
Aaron H. Gardner, MD, ${ }^{a}$ Parthak Prodhan, MD, ${ }^{a}$ Stephanie H. Stovall, MD, ${ }^{b}$ Jeffrey M. Gossett, MS, ${ }^{a}$ \\ Jennie E. Stern, BS, ${ }^{a}$ Christopher D. Wilson, RN, ${ }^{c}$ and Richard T. Fiser, MD ${ }^{\mathrm{a}}$
}

\begin{abstract}
Objective: Infections acquired by children during extracorporeal membrane oxygenation (ECMO) increase mortality. Our aim was to evaluate the effectiveness of prophylactic fluconazole on the incidence of fungal infections and to assess whether hospital-acquired fungal infection is associated with increased in-hospital mortality in pediatric cardiac patients requiring ECMO.
\end{abstract}

\begin{abstract}
Methods: We retrospectively reviewed a prospectively maintained database and collected data on all hospitalacquired infections in patients supported for cardiac indications at a tertiary children's hospital from 1989 to 2008.
\end{abstract}

\begin{abstract}
Results: ECMO was deployed 801 times in 767 patients. After exclusion criteria were applied, 261 pediatric patients supported for cardiac indications were studied. Fungal infection (blood, urine, or surgical site) occurred in $12 \%(31 / 261)$ of patients, $9(7 \%)$ of 127 patients receiving fluconazole prophylaxis versus $22(16.4 \%)$ of 134 without antifungal prophylaxis $(P=.02)$. Using a multivariable logistic regression model, the absence of fluconazole prophylaxis was associated with an increased risk of fungal infection (odds ratio $[\mathrm{OR}]=2.8 ; 95 \%$ confidence intervals $[\mathrm{CI}], 1.2,6.7 ; P=.016)$. In a multivariable logistic regression model for in-hospital mortality, the presence of fungal infection was associated with increased odds $(\mathrm{OR}=3.8 ; 95 \% \mathrm{CI}, 1.5,9.6 ; P=.005)$ of inhospital mortality among cardiac patients requiring ECMO, and the absence of antifungal prophylaxis showed a trend toward the same $(\mathrm{OR}=1.6 ; 95 \% \mathrm{CI}, 0.96,2.8 ; P=.072)$.
\end{abstract}

Conclusions: Children with cardiac disease supported with ECMO who acquire fungal infections have increased mortality. Routine fluconazole prophylaxis is associated with lower rates of fungal infections in these patients. (J Thorac Cardiovasc Surg 2012;143:689-95)

Extracorporeal membrane oxygenation (ECMO) is used to support cardiopulmonary function in patients with refractory but potentially reversible cardiac and/or respiratory failure. The risk of hospital-acquired infection (HAI) is substantial in both pediatric and adult patients supported with ECMO, including patients supported with ECMO after cardiotomy. ${ }^{1-11}$ In a 1996 review of data from the Extracorporeal Life Support Organization (ELSO) registry, Douglass, Keenan, and Purhoit ${ }^{9}$ highlighted the increased risk of hospital mortality in pediatric ECMO patients in whom infections developed. This increased risk has been documented in both retrospective and prospective studies. ${ }^{3,7}$ In the review of ELSO registry data, fungal

\footnotetext{
From the Department of Pediatrics, ${ }^{\text {a }}$ University of Arkansas for Medical Sciences, Arkansas Children's Hospital, Little Rock, Ark; the Children's Hospital of Southwest Florida, ${ }^{\text {b }}$ Lee Memorial Health System, Fort Myers, Fla; and the ECMO Team, ${ }^{\text {c }}$ Arkansas Children's Hospital, Little Rock, Ark.

This study was supported in part by the University of Arkansas for Medical Sciences College of Medicine Children's University Medical Group Fund Grant Program.

Disclosures: Authors have nothing to disclose with regard to commercial support.

Received for publication May 19, 2011; revisions received Sept 8, 2011; accepted for publication Dec 1, 2011; available ahead of print Dec 19, 2011.

Address for reprints: Richard T. Fiser, MD, University of Arkansas for Medical Sciences, Arkansas Children's Hospital, 1 Children's Way, Slot \# 512-12, Little Rock, AR 72202-3591 (E-mail: FiserRichard@uams.edu).

$0022-5223 / \$ 36.00$

Copyright (C) 2012 by The American Association for Thoracic Surgery doi:10.1016/j.jtcvs.2011.12.001
}

infections were uncommon $(0.6 \%)$ but were associated with higher hospital mortality rates than were bacterial infections. ${ }^{9}$ HAIs, particularly fungal infections, are an important source of added morbidity and mortality in critically ill patients supported with ECMO. ${ }^{1,2,9,12,13}$ In a recent study of HAI in more than 20,000 ECMO cases from the ELSO registry, Candida species were the second most common organism isolated. ${ }^{14}$

Two previous studies from our institution have examined HAI during ECMO support. ${ }^{1,2}$ In a retrospective analysis of 109 patients, Schutze and Heulitt ${ }^{1}$ demonstrated that infections developed during ECMO in 16\% of patients, of which $50 \%$ were due to fungal organisms. In 2001 , O'Neil and colleagues $^{2}$ re-examined the incidence and types of infections occurring during ECMO at our institution and found a $26 \%$ rate of HAI, with $38 \%$ being caused by fungal organisms. Urinary tract infections caused by fungal organisms were noted to be particularly prevalent. The first of these studies included 37 (34\%) of 109 and the second 75 $(53 \%)$ of $141(53 \%)$ pediatric cardiac patients requiring ECMO. ${ }^{1,2}$ As a result of the high incidence of fungal infection reported in these studies, a practice change was instituted to encourage the use of prophylactic fluconazole for pediatric patients supported with ECMO at our institution, particularly cardiac patients requiring ECMO. Although fluconazole has been rarely associated with 


\begin{tabular}{|ll|}
\hline \multicolumn{2}{|c|}{ Abbreviations and Acronyms } \\
$\mathrm{CI} \quad=$ & confidence intervals \\
$\mathrm{ECMO}=$ & extracorporeal membrane \\
& oxygenation \\
ELSO $=$ & Extracorporeal Life Support \\
& Organization \\
$\mathrm{HAI}$ & $=$ hospital-acquired infection \\
$\mathrm{IQR}$ & $=$ interquartile range \\
$\mathrm{OR}$ & $=$ odds ratio \\
RACHS-1 & Risk Adjustment for Congenital \\
& Heart Surgery \\
ROC & receiver operating characteristic \\
&
\end{tabular}

hepatotoxicity and cholestasis, large studies of prophylaxis with fluconazole to prevent invasive fungal infections have found it to be both safe and efficacious in such populations as premature and low-birth-weight infants and immunocompromised patients. ${ }^{15-17}$ The effectiveness of this practice has not been previously evaluated in pediatric patients supported with ECMO. The primary aim of this study was to evaluate the effect of prophylactic fluconazole therapy on the incidence of fungal infections in pediatric cardiac patients supported with ECMO. A secondary aim was to evaluate the impact of extrapulmonary fungal HAI on in-hospital mortality in this population.

\section{PATIENTS AND METHODS \\ Setting and Study Population}

This study was approved by the Institutional Review Board at the University of Arkansas for Medical Sciences, and the requirement for informed consent was waived. This study was conducted at Arkansas Children's Hospital, a 316-bed free-standing tertiary children's hospital in Little Rock, Arkansas. We performed a retrospective review of an institutional ECMO database and identified 801 ECMO deployments provided to 767 patients from March 1989 through July 2008. We excluded all patients 19 years of age or older at the initiation of ECMO support, patients with ECMO duration of less than 48 hours, patients with incomplete microbiologic data, and patients supported with ECMO for respiratory failure (Figure 1). We also excluded patients transported to other institutions by our mobile ECMO team. After exclusion criteria were applied, 261 cardiac patients supported by ECMO were studied.

\section{Data Collection}

Data were obtained by reviewing a prospectively maintained institutional ECMO database and hospital medical records, including physician orders, daily progress notes, laboratory records, pharmacy records, and daily ECMO records maintained by ECMO specialists and coordinators. Data collected included patient demographics, diagnoses at initiation of ECMO support, duration of ECMO, use of fluconazole prophylaxis, any positive culture between the initiation of ECMO and 7 days after ECMO, broad-spectrum antibiotic use during ECMO support, operative status (postoperative/bridged to transplant, postoperative/not bridged to transplant, not postoperative/bridged to transplant, not postoperative/not bridged to transplant, or cardiac transplant), Risk Adjustment for
Congenital Heart Surgery (RACHS-1) score, ${ }^{18}$ and survival to hospital discharge. For patients with more than 1 episode of ECMO support during a hospitalization, only the first episode was analyzed.

\section{Study Definitions}

For purposes of this study, the patient was considered to have received fluconazole prophylaxis if no pre-existing fungal infection was identified and if intravenous fluconazole therapy was initiated within the first 24 hours of ECMO support. Prophylactic fluconazole therapy has been a component of the standard ECMO order set at our institution since the late $1990 \mathrm{~s}$, but with its use left at the discretion of the patient's physician. Intravenous fluconazole is administered at a dose of $6 \mathrm{mg} / \mathrm{kg}$ once daily, with dosing decreased $50 \%$ for significant renal impairment. Prophylactic fluconazole treatment is discontinued at the time of discontinuation of ECMO support unless the patient has a documented fungal infection requiring further treatment. Routine surveillance cultures of blood, urine, tracheal secretions, and surgical sites are obtained daily for all pediatric patients supported with ECMO at our institution.

A positive culture was defined as recovery of an organism from blood, urine, or surgical site not identified before ECMO support but collected 48 hours or more after hospital admission. Positive cultures within the first 48 hours of hospitalization were excluded to prevent inclusion of patients with community-acquired infections. A HAI was defined as an infection that was not present, nor an extension of an infection present, on admission to the hospital. ${ }^{19}$ All HAIs while the patient was receiving ECMO support or within 7 days after ECMO were recorded.

Microbiologic records were screened for the following fungal organisms: Candida spp (albicans, tropicalis, glabrata, parapsilosis, krusei, and lusitaniae), Aspergillus spp (fumigatus, flavus, and niger), Blastomyces, Coccidioides, Histoplasma, Cryptococcus neoformans, and Trichosporon spp (belgelii and cutaneum). Extrapulmonary fungal HAIs (from blood, urine, or surgical sites) were considered a clinically significant outcome for analysis. Positive endotracheal cultures for fungal organisms were not analyzed for the following reasons: (1) the technique for obtaining pulmonary secretions for culture at our institution was not consistent during the study period or (2) the difficulty in delineating fungal colonization of an endotracheal tube from a true ventilator-associated pneumonia in pediatric patients. ${ }^{20}$

We recorded the use of extended-spectrum penicillins and third- and fourth-generation cephalosporins before the first positive culture. Once a positive culture was identified, pharmacy records were examined to determine whether these broad-spectrum antibiotics were used before the infection and, if so, the duration of therapy.

\section{Statistical Analysis}

Continuous variables were summarized using the median and interquartile range (IQR). The IQR is the interval from the first quartile to the third quartile (25th-75th percentile). Discrete variables were summarized as frequency and percentage. Characteristics of patients were compared by treatment group (prophylactic fluconazole versus control) using the Wilcoxon rank sum tests for continuous variables and Pearson $\chi^{2}$ tests for categorical variables (Table 1). Multivariable logistic regression analysis was used to evaluate the association between the candidate independent variables and the outcomes fungal infection and death during the hospitalization. The independent candidate predictor variables in the model included ECMO duration (indicator for $>10$ days), age at initiation of ECMO support (indicator for $<30$ days), treatment with prophylactic fluconazole, operative status ( 5 categories: postoperative/bridged to transplant, postoperative/not bridged to transplant, not postoperative/bridged to transplant, not postoperative/not bridged to transplant, cardiac transplant), and history of delayed sternal closure. The model for hospital mortality was fit with and without an indicator variable for fungal infection. The area under the receiver operating 


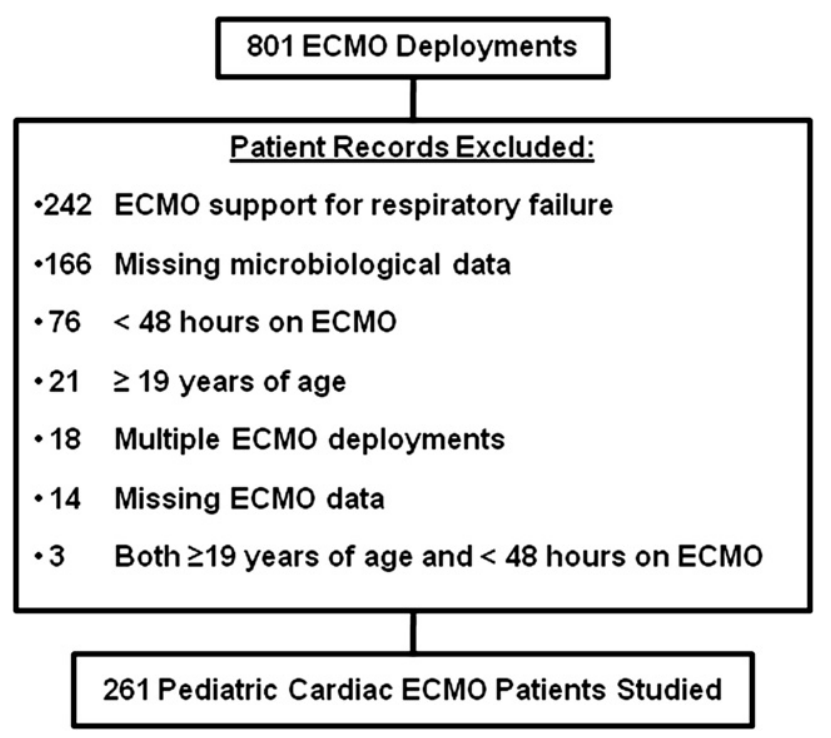

FIGURE 1. Flow chart showing inclusion and exclusion criteria. ECMO, Extracorporeal oxygenation.

characteristic (ROC) curve was used to quantify model discrimination. A validated area under the ROC was calculated using 100 bootstrap samples. The validated ROC estimates the performance of the model on other data sets. The Hosmer-Lemeshow goodness-of-fit test was used to evaluate model calibration. Stata/MP11 (Stata Corporation, College Station, Tex) and the Harrell regression modeling strategies package in $\mathrm{R}$ were used for the analyses.

\section{RESULTS \\ Patient Characteristics}

A total of 767 patients underwent 801 ECMO deployments for respiratory or cardiac support from 1989 to 2008. Of the 801 cases, 540 were excluded, leaving 261 patients supported with ECMO for cardiac indications for analysis (Figure 1). A description of the study sample is shown in Table 2.

\section{Fungal Infection}

The incidence of extrapulmonary fungal $\mathrm{HAI}$ in pediatric cardiac patients requiring ECMO was 12\% (31/261). Sites for fungal infection included surgical site $(5 \%, 13 / 261)$, urine $(5 \%, 15 / 261)$, and bloodstream 3.4\% (9/261). Extrapulmonary fungal HAI occurred in 7\% (9/127) of patients receiving fluconazole prophylaxis versus $16.4 \%(22 / 134)$ without antifungal prophylaxis $(P=.02)$ (Table 1). Fungemia occurred in $2.4 \%$ (3/127) of patients receiving fluconazole prophylaxis versus $4.5 \%(6 / 134)$ of patients not receiving antifungal prophylaxis ( $P=$ not significant). Eighty-seven percent (27/31) of patients had positive fungal cultures from more than 1 site (blood, urine, or surgical site). Candida species accounted for $29(94 \%)$ of 31 positive fungal cultures, with the remainder being Aspergillus species.

Longer duration of ECMO support was associated with fungal HAI. Median (IQR) ECMO duration (hours) was $168(95,263)$ in the group with negative fungal cultures

TABLE 1. Demographic comparison by fungal infection

\begin{tabular}{|c|c|c|c|c|}
\hline \multirow[b]{2}{*}{ Characteristics } & \multicolumn{3}{|c|}{ Fungal infection } & \multirow[b]{2}{*}{$P$ value } \\
\hline & No & Yes & Total & \\
\hline No. of patients & 230 & 31 & 261 & \\
\hline Male, n (\%) & $129(56 \%)$ & $14(45 \%)$ & $143(55 \%)$ & .25 \\
\hline \multicolumn{5}{|l|}{ Age, mo } \\
\hline Median (IQR) & $2(0.03,14)$ & $2(0.4,18)$ & $2(0.03,14)$ & .36 \\
\hline$<30$ days, $\mathrm{n}(\%)$ & $104(45 \%)$ & $13(42 \%)$ & $117(45 \%)$ & .73 \\
\hline \multicolumn{5}{|l|}{ Duration of ECMO, $h$} \\
\hline Median (IQR) & $168(95,263)$ & $236(180,336)$ & $175(108,272)$ & .001 \\
\hline$\geq 240$ h, n (\%) & $167(29 \%)$ & $15(48 \%)$ & $82(31 \%)$ & .03 \\
\hline \multicolumn{5}{|l|}{ Mode of ECMO } \\
\hline Venoarterial, n (\%) & $224(97 \%)$ & $31(100 \%)$ & $255(98 \%)$ & .36 \\
\hline Operative status, n (\%) & & & & .80 \\
\hline Postoperative, bridged to transplant & $15(7 \%)$ & $1(3 \%)$ & $16(6 \%)$ & \\
\hline Postoperative, not bridged to transplant & $120(52 \%)$ & $19(61 \%)$ & $139(53 \%)$ & \\
\hline Not postoperative, bridged to transplant & $32(14 \%)$ & $5(16 \%)$ & $37(14 \%)$ & \\
\hline Not postoperative, not bridged to transplant & $52(23 \%)$ & $5(16 \%)$ & $57(22 \%)$ & \\
\hline Cardiac transplant & $11(5 \%)$ & $1(3 \%)$ & $12(5 \%)$ & \\
\hline Delayed sternal closure, n (\%) & $130(57 \%)$ & $19(61 \%)$ & $149(57 \%)$ & .61 \\
\hline Antifungal prophylaxis, n (\%) & $118(51 \%)$ & $9(29 \%)$ & $127(49 \%)$ & .02 \\
\hline Death before discharge, $\mathrm{n}(\%)$ & $101(44 \%)$ & $24(77 \%)$ & $125(48 \%)$ & $<.001$ \\
\hline ECMO era, $\mathrm{n}(\%)$ & & & & .001 \\
\hline Started on ECMO 1989-2001 & $99(43 \%)$ & $23(74 \%)$ & $122(47 \%)$ & \\
\hline Started on ECMO 2002-2008 & $131(57 \%)$ & $8(26 \%)$ & $139(53 \%)$ & \\
\hline
\end{tabular}


TABLE 2. Description of the study sample by prophylactic antifungal therapy

\begin{tabular}{|c|c|c|c|c|}
\hline \multirow[b]{2}{*}{ Characteristics } & \multicolumn{3}{|c|}{ Prophylactic antifungal therapy } & \multirow[b]{2}{*}{$P$ value } \\
\hline & No & Yes & Total & \\
\hline No. of patients & 134 & 127 & 261 & \\
\hline Male, n $(\%)$ & $67(50 \%)$ & $76(60 \%)$ & $143(55 \%)$ & .11 \\
\hline \multicolumn{5}{|l|}{ Age, mo } \\
\hline Median (IQR) & $1.4(0.033,17)$ & $2.4(0.033,14)$ & $2.0(0.033,14)$ & .76 \\
\hline$<30$ days, $\mathrm{n}(\%)$ & $61(46 \%)$ & $56(44 \%)$ & $117(45 \%)$ & .82 \\
\hline \multicolumn{5}{|l|}{ Duration of ECMO, $\mathrm{h}$} \\
\hline Median (IQR) & $171(111,257)$ & $177(100,286)$ & $175(108,272)$ & .74 \\
\hline$\geq 240 \mathrm{~h}, \mathrm{n}(\%)$ & $37(28 \%)$ & $45(35 \%)$ & $82(31 \%)$ & .17 \\
\hline \multicolumn{5}{|l|}{ Mode of ECMO } \\
\hline Venoarterial, n (\%) & $224(97 \%)$ & $31(100 \%)$ & $255(98 \%)$ & .36 \\
\hline Operative status, $\mathrm{n}(\%)$ & & & & .12 \\
\hline Postoperative, bridged to transplant & $8(6 \%)$ & $8(6 \%)$ & $16(6 \%)$ & \\
\hline Postoperative, not bridged to transplant & $78(58 \%)$ & $61(48 \%)$ & $139(53 \%)$ & \\
\hline Not postoperative, bridged to transplant & $12(9 \%)$ & $25(20 \%)$ & $37(14 \%)$ & \\
\hline Not postoperative, not bridged to transplant & $31(23 \%)$ & $26(20 \%)$ & $57(22 \%)$ & \\
\hline Cardiac transplant & $5(4 \%)$ & $7(6 \%)$ & $12(5 \%)$ & \\
\hline RACHS-1 classification, $\mathrm{n}(\%)$ & & & & .58 \\
\hline Not applicable & $49(37 \%)$ & $59(46 \%)$ & $108(41 \%)$ & \\
\hline 1 & $0(0 \%)$ & $1(1 \%)$ & $1(0 \%)$ & \\
\hline 2 & $6(4 \%)$ & $4(3 \%)$ & $10(4 \%)$ & \\
\hline 3 & $32(24 \%)$ & $26(20 \%)$ & $58(22 \%)$ & \\
\hline 4 & $24(18 \%)$ & $16(13 \%)$ & $40(15 \%)$ & \\
\hline 5 & $2(1 \%)$ & $1(\% 1)$ & $3(1 \%)$ & \\
\hline 6 & $21(16 \%)$ & $20(16 \%)$ & $41(16 \%)$ & \\
\hline Delayed sternal closure, n (\%) & $81(60 \%)$ & $68(54 \%)$ & $149(57 \%)$ & .26 \\
\hline Death before discharge, $\mathrm{n}(\%)$ & $73(54 \%)$ & $52(41 \%)$ & $125(48 \%)$ & .029 \\
\hline ECMO era, $\mathrm{n}(\%)$ & & & & $<.001$ \\
\hline Started on ECMO 1989-2001 & $113(84 \%)$ & $9(7 \%)$ & $122(47 \%)$ & \\
\hline Started on ECMO 2002-2008 & $21(16 \%)$ & $118(93 \%)$ & $139(53 \%)$ & \\
\hline
\end{tabular}

$P$ values based on $\chi^{2}$ tests for categorical variables or the Wilcoxon rank sum test for continuous variables. IQR, Interquartile range (25th percentile, 75 th percentile); $E C M O$, extracorporeal membrane oxygenation; RACHS-1, Risk Adjustment for Congenital Heart Surgery.

versus $236(180,336)$ in the group in which a positive fungal culture developed $(P<.001)$. Of those patients with a fungal infection, $35 \%$ (11/31) had been previously treated with broad-spectrum antibiotics; 5 with third- or fourthgeneration cephalosporins, 4 with extended-spectrum penicillins, and 2 with both.

\section{Fluconazole Therapy and Risk of Fungal Infection}

Patients who received prophylactic fluconazole were similar to controls on baseline measures (Table 2). In a multivariable logistic regression model to predict fungal infection, the absence of fluconazole prophylaxis was associated with an increased risk of fungal infection (odds ratio $[\mathrm{OR}]=2.8$; $95 \%$ confidence intervals [CI] $1.2,6.7 ; P=.016$ ) (Table 3). Neither neonatal age nor delayed sternal closure was associated with a risk of fungal infection in this model.

\section{Fungal Infection and In-Hospital Mortality}

Fungal infection was associated with increased mortality in pediatric cardiac patients requiring ECMO. Of 31 patients with fungal infections, $24(77 \%)$ did not survive to hospital discharge. With the same preselected, clinically relevant variables, a multivariable logistic regression model to predict in-hospital mortality demonstrated that a fungal infection of blood, urine, or surgical site was associated with an increased risk of in-hospital mortality $(\mathrm{OR}=3.8$; $95 \% \mathrm{CI}, 1.5,9.6 ; P=.005)$. Absence of prophylactic fluconazole use did not attain statistical significance in a model also containing fungal infection $(\mathrm{OR}=1.6 ; 95 \% \mathrm{CI}$, $0.96,2.8 ; P=.07)$. Given that our model contains both treatment with fluconazole and fungal infection, the effect of fluconazole is mediated. In a model that omits fungal infection, the absence of prophylactic fluconazole does attain statistical significance $(\mathrm{OR}=1.8 ; 95 \% \mathrm{CI}, 1.3,3.1$; $P=.023)$. Other variables in the model that were associated with an increased risk of hospital mortality were neonatal age, delayed sternal closure, and duration of ECMO support greater than 10 days (Table 4).

\section{DISCUSSION}

Our study is the first to examine the potential effectiveness of prophylactic fluconazole to prevent fungal HAIs 
TABLE 3. Multivariable logistic regression analysis for predictors of fungal infection

\begin{tabular}{lcc}
\hline \multicolumn{1}{c}{ Variables } & OR $(\mathbf{9 5} \% \mathbf{C I})$ & $\begin{array}{c}\boldsymbol{P} \\
\text { value }\end{array}$ \\
\hline Age $<30 \mathrm{~d}$ & $0.73(0.32,1.6)$ & .44 \\
Delayed sternal closure & $0.9(0.26,3.4)$ & .93 \\
Operative status & & \\
$\quad$ Postoperative, bridged to transplant & 1 & \\
$\quad$ Postoperative, not bridged to transplant & $3.5(0.41,30)$ & .25 \\
Not postoperative, bridged to transplant & $2.9(0.23,36)$ & .41 \\
$\quad$ Not postoperative, not bridged to transplant & $2.4(0.2,30)$ & .49 \\
Cardiac transplant & $2.1(0.1,42)$ & .64 \\
ECMO duration $\geq 240 \mathrm{~h}$ & $2.8(1.2,6.5)$ & .017 \\
No antifungal prophylaxis & $2.8(1.2,6.7)$ & .016 \\
\hline
\end{tabular}

Validated Area under the receiver operating characteristic is 0.59 . Hosmer-Lemeshow goodness-of-fit test, $P=.20$, which is consistent with adequate fit. $O R$, Odds ratio; $C I$, confidence interval; $E C M O$, extracorporeal membrane oxygenation.

in pediatric cardiac patients requiring ECMO. Prophylactic fluconazole therapy was associated with a decreased incidence of fungal infections from blood, urine, or surgical sites, with patients not receiving prophylactic fluconazole having a 2.8 -fold increased risk of having a fungal HAI develop. The use of prophylactic fluconazole was associated with a strong trend toward decreased mortality. Development of an extrapulmonary fungal HAI in pediatric cardiac patients requiring ECMO was associated with a 3.8-fold increased risk of in-hospital mortality. In addition to the use of prophylactic fluconazole, the other clinical variable significantly associated with a lower risk of fungal infection was a shorter duration of ECMO support.

To our knowledge, this is the first study to specifically describe the impact of extrapulmonary fungal HAI on inhospital mortality in this patient population. Previous work at our institution identified major surgical procedures before or during ECMO support, as well as transthoracic ECMO cannulation and delayed sternal closure, as significant risk factors for any type of HAI. ${ }^{2}$ In the current study, however, delayed sternal closure was not associated with an increased rate of fungal HAI. This finding is somewhat surprising and is in contrast to a recent study demonstrating that a multidisciplinary team aimed at decreasing the rate of health care-associated infections in patients requiring ECMO was able to decrease the rate of sepsis in part by decreasing the number of patients with open chest cannulation. $^{3}$

Patients receiving ECMO for cardiac support have several reasons for being at increased risk of HAI in general and from fungal infections specifically. Significant risk factors for any type of HAI include prolonged duration of ECMO support-particularly in patients awaiting heart transplantation, breach in anatomic host barrierpre-ECMO or post-ECMO, major surgical procedures, transthoracic ECMO cannulation, and delayed sternal closure. ${ }^{2}$ Cardiac patients receiving ECMO support may also have relative immunodeficiency: preoperative and postoperative absolute lymphopenia, cardiopulmonary bypassassociated/induced monocyte deactivation, or genetic syndromes that themselves predispose to immune deficiency. $^{21-24}$ One may speculate that a combination of these factors might predispose cardiac patients requiring ECMO to an increased risk of fungal infection and may make them more likely to benefit from antifungal prophylaxis while on ECMO support.

It is not surprising that a high percentage of patients (approximately one third) in whom a fungal infection developed during ECMO support had received broad-spectrum antibiotics, specifically third- or fourth-generation cephalosporins, before a fungal infection developed. Broadspectrum antibiotics such as these have been documented in a variety of clinical situations to suppress normal flora and promote overgrowth of fungal species, such as Candida spp.

Patients in whom fungal infections developed required a longer duration of ECMO support than those who did not, with a difference of approximately 1 day in the mean

TABLE 4. Adjusted effects of multivariable logistic regression analysis for predictors of in-hospital mortality

\begin{tabular}{|c|c|c|c|c|}
\hline \multirow[b]{2}{*}{ Variables } & \multicolumn{2}{|c|}{ All regressors } & \multicolumn{2}{|c|}{ Fungal infection omitted } \\
\hline & OR $(95 \%$ CI $)$ & $P$ value & OR $(95 \%$ CI $)$ & $P$ value \\
\hline Age $<30 \mathrm{~d}$ & $2.1(1.2,3.6)$ & .01 & $2.0(1.1,3.4)$ & .009 \\
\hline Delayed sternal closure & $1.7(0.71,3.9)$ & .25 & $1.6(0.7,3.8)$ & .25 \\
\hline \multicolumn{5}{|l|}{ Operative status } \\
\hline Postoperative, bridged to transplant & 1 & & 1 & \\
\hline Postoperative, not bridged to transplant & $0.97(0.33,3.2)$ & .95 & $1.2(0.4,3.7)$ & .74 \\
\hline Not postoperative, bridged to transplant & $0.71(0.17,3)$ & .64 & $0.83(0.2,3.4)$ & .79 \\
\hline Not postoperative, not bridged to transplant & $1.1(0.28,4.6)$ & .85 & $1.3(0.33,5.1)$ & .72 \\
\hline Cardiac transplant & $1.2(0.22,6.8)$ & .81 & $1.4(0.25,7.7)$ & .70 \\
\hline ECMO duration $\geq 240 \mathrm{~h}$ & $2.6(1.4,4.9)$ & .004 & $2.9(1.6,5.4)$ & .001 \\
\hline No antifungal prophylaxis & $1.6(0.96,2.8)$ & .07 & $1.8(1.1,3.1)$ & .023 \\
\hline Fungal infection (extrapulmonary) & $3.8(1.5,9.6)$ & .005 & Omitted & \\
\hline
\end{tabular}

Validated area under the receiver operating characteristic is 0.68 for full model and 0.63 for model omitting fungal infection. Both models had adequate fit based on HosmerLemeshow goodness-of-fit test. $O R$, Odds ratio; $C I$, confidence interval; $E C M O$, extracorporeal membrane oxygenation. 
support time between the 2 groups. As would be expected, a recent review of the ELSO registry showed that the risk of any HAI increases with the duration of invasive support such as ECMO. ${ }^{14}$

\section{Study Limitations}

This study has several limitations. First, as a retrospective study, it is subject to reporting bias and is also subject to potential inconsistencies or gaps in data reviewed for the study. As mentioned previously, approximately 300 potential study patients had to be excluded. The majority of patient exclusions were due to missing microbiologic data from the era in which records were paper-based (before 1998). However, the remaining patients, with complete data sets, represent a vast clinical experience at a large ECMO center over a period of 2 decades. Another potential limitation is the fact that prophylactic fluconazole use for a patient requiring ECMO at our institution is at the discretion of the attending physician. Thus, our study could potentially be biased toward "sicker" patients receiving fluconazole or from other biases in individual physician practice. However, as noted in Table 2, 93\% (118) of pediatric cardiac patients on ECMO treated between 2002 and 2008 received antifungal prophylaxis and, again, the large number of patients evaluated decreases the chance that the primary findings are due to bias. The study did not aim to specifically determine any "break points" in ECMO duration as related to risk of fungal infection, but such analysis may prove beneficial in the future for improved targeting of prophylactic fluconazole use, particularly in cardiac patients requiring ECMO. We also recognize that numerous other factors may influence infection and mortality in patients requiring ECMO and that many advances in the management of these patients have occurred concurrently with the introduction of routine antifungal prophylaxis at our institution. Quantifying and controlling for all these variables is well beyond the scope of our study. However, the significance of fungal HAI in patients requiring ECMO, and the high risk of mortality that accompanies them, warrants our reporting of the data.

\section{CONCLUSIONS}

In pediatric patients supported with ECMO for cardiac indications, the presence of fungal infection increases the risk of in-hospital mortality and routine fluconazole prophylaxis is associated with a decreased incidence of fungal infections. The absence of prophylactic fluconazole therapy was associated with increased mortality. To our knowledge, this is the first study to demonstrate the effectiveness of antifungal prophylaxis in children during ECMO for cardiac support. Further prospective investigation into the use of antifungal prophylaxis in patients requiring ECMO is warranted.
We gratefully acknowledge Dr Stephen M. Schexnayder and Dr Todd G. Nick for their review of this manuscript and the dedication, hard work, and exceptional care provided daily by the ECMO team at Arkansas Children's Hospital.

\section{References}

1. Schutze GE, Heulitt MJ. Infections during extracorporeal life support. J Pediatr Surg. 1995;30:809-12.

2. O'Neil JM, Schutze GE, Heulitt MJ, Simpson PM, Taylor BJ. Nosocomial infections during extracorporeal membrane oxygenation. Int Care Med. 2001;27: 1247-53.

3. Brown KL, Ridout DA, Shaw M, Dodkins I, Smith LC, O'Callaghan MA, et al. Healthcare-associated infection in the pediatric patients on extracorporeal life support: the role of multidisciplinary surveillance. Pediatr Crit Care Med. 2006; 7:546-50

4. Steiner CK, Stewart DL, Bond SJ, Hornung CA, McKay VJ. Predictors of acquiring a nosocomial bloodstream infection on extracorporeal membrane oxygenation. J Pediatr Surg. 2001;36:487-92.

5. Elerian LF, Sparks JW, Meyer TA, Zwischenberger JB, Doski J, Goretsky MJ, et al. Usefulness of surveillance cultures in neonatal extracorporeal membrane oxygenation. ASAIO J. 2001;47:220-3.

6. Burket JS, Bartlett RH, Vander Hyde K, Chenoweth CE. Nosocomial infections in adult patients undergoing extracorporeal membrane oxygenation. Clin Infect Dis. 1999;28:828-33.

7. Montgomery VL, Strotman JM, Ross MP. Impact of multiple organ system dysfunction and nosocomial infections on survival of children treated with extracorporeal membrane oxygenation after heart surgery. Crit Care Med. 2000;28: 526-31.

8. Coffin SE, Bell LM, Manning M, Polin R. Nosocomial infections in neonates receiving extracorporeal membrane oxygenation. Infect Control Hosp Epidemiol. 1997; 18:93-6.

9. Douglass BH, Keenan AL, Purhoit DM. Bacterial and fungal infection in neonates undergoing venoarterial extracorporeal membrane oxygenation: an analysis of the registry data of the extracorporeal life support organization. Artif Organs. 1996;20:202-8.

10. Meyer DM, Jesson ME, Eberhart RC, the Extracorporeal Life Support Organization. Neonatal extracorporeal membrane oxygenation complicated by sepsis. Ann Thorac Surg. 1995;59:975-80.

11. Kumar TKS, Zurakowski D, Dalton H, Talwar S, Allard-Picou A, Duebener LF, et al. Extracorporeal membrane oxygenation in postcardiotomy patients: factors influencing outcome. J Thorac Cardiovasc Surg. 2010;140: 330-336.e2

12. Sun H-Y, Ko W-J, Tsai P-R, Sun C-C, Chang Y-Y, Lee C-W, et al. Infections occurring during extracorporeal membrane oxygenation use in adult patients. J Thorac Cardiovasc Surg. 2010;140:1125-1132.e2.

13. Hoskote A, Bohn D, Gruenwald C, Edgell D, Cai S, Adatia I, et al. Extracorporeal life support after staged palliation of a functional single ventricle: subsequent morbidity and survival. J Thorac Cardiovasc Surg. 2006;131: 1114-21.

14. Bizzarro MJ, Conrad SA, Kaufman DA, Rycus P. Extracorporeal Life Support Organization Task Force on Infections, Extracorporeal Membrane Oxygenation. Infections acquired during extracorporeal membrane oxygenation in neonates, children, and adults. Pediatr Crit Care Med. 2011;12:277-81.

15. Martin A, Pappas A, Lulic-Botica M, Natarajan G. Impact of "targeted" fluconazole prophylaxis for preterm neonates: efficacy of a highly selective approach? J Perinatol. 2011 Jul 14; 10.1038/jp.2011.27 [Epub ahead of print].

16. Pechlivanoglou P, Vries RD, Daenen SM, Postma MJ. Cost benefit and cost effectiveness of antifungal prophylaxis in immunocompromised patients treated for haematological malignancies: reviewing the available evidence. Pharmacoeconomics. 2011;29:737-51

17. Manzoni P, Jacqz-Aigrain E, Rizzollo S, Franco C, Stronati M, Mostert M, et al. Antifungal prophylaxis in neonates. Early Hum Dev. 2011 Mar;87(Suppl. 1): S59-60.

18. Jenkins KJ, Gauvreau K, Newburger JW, Spray TL, Moller JH, Iezzoni LI. Consensus-based method for risk adjustment for surgery for congenital heart disease. J Thorac Cardiovasc Surg. 2002;123:110-8.

19. Horan TC, Andrus M, Dudeck MA. CDC/NHSN surveillance definition of health care-associated infection and criteria for specific types of infections in the acute care setting. Am J Infect Control. 2008;36:309-32. 
20. Mayhall CG. Ventilator-associated pneumonia or not? Contemporary diagnosis. Emerg Infect Dis. 2001;7:200-4.

21. Fiser WP, Yetman AT, Gunselman RJ, Fasules JW, Baker LL, Chipman CW, et al. Pediatric arteriovenous extracorporeal membrane oxygenation (ECMO) as a bridge to cardiac transplantation. J Heart Lung Transplant. 2003;22: 770-7.

22. Cabrera AG, Dyamenahalli U, Gossett J, Prodhan P, Morrow WR, Imamura M, et al. Preoperative lymphopenia is a predictor of postoperative adverse outcomes in children with congenital heart disease. J Thorac Cardiovasc Surg. 2009;138 1172-9.

23. Shi SS, Shi CC, Zhao ZY, Shen HQ, Fang XM, Tan LH, et al. Effect of open hear surgery with cardiopulmonary bypass on peripheral blood lymphocyte apoptosis in children. Pediatr Cardiol. 2009;30:153-9.

24. Allen ML, Peters MJ, Goldman A, Elliott M, James I, Callard R, et al. Early postoperative monocyte deactivation predicts systemic inflammation and prolonged stay in pediatric cardiac intensive care. Crit Care Med. 2002;30:1140-5. 